\title{
REDUCED MODULES ARE ABELIAN
}

\author{
${ }^{1}$ Eunike Irene Kumaseh. ${ }^{2}$ Yohanes A. R. Langi \\ ${ }^{1}$ Mathematics De partment, Sam Ratulangi Uni versity, Manado, eunikekumaseh@rocketmail.com \\ ${ }^{2}$ Mathematics Department, Sam Ratulangi Uni versity, Manado, yarlangi@gmail.com
}

\begin{abstract}
In this research that introduce abelian modules as a generalization of abelian rings. Let $R$ be an arbitrary ring with identity 1 . A module $M$ is called reduced if, for any $\boldsymbol{m} \in \boldsymbol{M}$ and any $\boldsymbol{a} \in \boldsymbol{R}, \boldsymbol{m a}=\mathbf{0}$ implies $\boldsymbol{m} \boldsymbol{R} \cap \boldsymbol{M a}=\mathbf{0}$.

This research using study literatures, to prove that every reduced module is abelian. A module $M$ is called abelian if, for any $\mathrm{m} \in \mathrm{M}$ and any $\mathrm{a} \in \mathrm{R}$, any ide mpotent $\mathrm{e} \in \mathrm{R}$, mae $=$ mea.

If $x \in m R \cap M a$, then there exists $m_{1} \in M$ and $r_{1} \in R$ such that $x=m r_{1}=m_{1} a$. Since $M$ is a p.p.module, $m a=0$ implies that $a \in r_{R}(m)=e R$ for some idempotent $e^{2}=e \in R$. Then $a=e a$ and $x e=m r_{1} e=m_{1} a e$. Since $M$ is abelian and $m e=0, m r_{1} e=m e r_{1}=m_{1} a e=m_{1} e a=m_{1} a=0$. So, $x=m r_{1}=m_{1} a=0$. Hence $m R \cap M a=0$, that is, $M$ is reduced. Reduced modules are abelian.
\end{abstract}

Keywords : Reduced Modules, Abelian Modules

\section{RINGKAS AN}

Dalam penelitian ini memperkenalkan tentang modul-modul yang abelian sebagai generalisasi dari gelanggang-gelanggang yang abelian. Misalkan $\boldsymbol{R}$ sebarang gelanggang dengan Identitas 1. Sebuah modul $\boldsymbol{M}$ dikatakan "reduced" jika, untuk setiap $\boldsymbol{m} \in \boldsymbol{M}$ and setiap $\boldsymbol{a} \in \boldsymbol{R}, \boldsymbol{m a}=\mathbf{0}$ maka $\boldsymbol{m} \boldsymbol{R} \cap \boldsymbol{M} \boldsymbol{a}=$ 0.

Penelitian ini berupa studi literatur, untuk membuktikan setiap modul reduced adalah abelian. Sebuah modul $M$ dikatakan Abelian jika, untuk setiap $m \in M$ dan $a \in R$, ada idempoten $e \in R$, mae $=$ mea .

Jika $x \in m R \cap M a$, maka ada $m_{1} \in M$ dan $r_{1} \in R$ sehingga $x=m r_{1}=m_{1} a$. Sejak $M$ adalah sebuah p.p.-module, $m a=0$ implikasinya $a \in r_{R}(m)=e R$ untuk beberapa idempoten $e^{2}=e \in R$. Maka $a=e a$ dan $x e=m r_{1} e=m_{1} a e$.

Sejak $M$ adalah abelian dan $m e=0, m r_{1} e=m e r_{1}=m_{1} a e=m_{1} e a=m_{1} a=0$. Jadi, $x=m r_{1}=$ $m_{1} a=0$. Sehingga $m R \cap M a=0$, maka, $M$ reduced. Modul-modul reduced adalah abelian.

Kata Kunci: Modul-modul Reduced, modul-modul yang abelian.

\section{Introduction}

Throughout this paper $\mathrm{R}$ denotes an associative ring with identity 1 , and modules will be unitary right $\mathrm{R}$-modules.

A left $R$-module $M$ over the ring $R$ consists of an abe lian group $(M,+)$ and an operation $R \times M$ $\rightarrow M$ (called scalar multiplication, usually just written by juxtaposition, i.e. as $r x$ for $r$ in $R$ and $x$ in $M$ ) such that

For all $r, s$ in $R, x, y$ in $M$, we have

1. $r(x+y)=r x+r y$

2. $(r+s) x=r x+s x$

3. $(r s) x=r(s x)$

4. $1_{R} x=x$ if $R$ has multiplicative identity $1_{R}$.

Recall that a ring is reduced if it has no nonzero nilpotent elements. A module $M$ is called reduced if, for any $m \in M$ and any $a \in R, m a=0$ implies $m R \cap M a=0$. Let $e$ be an idempotent in $R$.

The ring $\mathrm{R}$ is called semicommutative if for any $a, b \in R, a b=0$ implies $a R b=0$. A module $M_{R}$ is called semicommutative if, for any $m \in M$ and any $a \in R, m a=0$ implies $m R a=0$ [1]. This problema Are reduced modules abelian? This research limited on Reduced modules. Objective of research to prove that every reduced module is abelian 


\section{Group}

Definition 1.

Let $\mathrm{G}$ be a nonempty set together with a binary operation (usually called multiplication) that assigns to each ordered pair $(a, b)$ of elements of $G$ an element in $G$ denoted by ab. We say $G$ is a group under this operation if the following three properties are satisfied.

1. Associativity. The operation is associative; that is, $(a b) c=a(b c)$ for all $a, b, c$ in $\mathrm{G}$.

2. Identity. There is an element $e$ (called the identity) in $G$, such that $a e=e a=a$ for all $a$ in G.

3. Inverses. For each element $a$ in $G$, there is an element $b$ in $G$ (called an inverse of $a$ ) such that $a b=b a=e$.

If a group has the property that $a b=b a$ for every pair of elements $a$ and $b$, we say the group is commutative or-better-yet-Abelian (in honor of the great Norwegian mathematician Niels Abel).

A group is non-Abelian if there is some pair of elements $a$ and $b$ for which $a b \neq b a$. When encountering a particular group for the first time, one should determine whether or not it is Abelian.

\section{Example 1.}

The set of integers $\mathbb{Z}$ (so denoted because the German word for integers is Zahlen), the set of rational numbers $\mathbb{Q}$ (for quotient), and the set of real numbers $\mathbb{R}$ are all groups under ordinary addition. In each case the identity is 0 and the inverse of $a$ is $-a$.

Definition 2.

(Group Homomorphism) A homomorphism $\phi$ from a group $G$ to a group $\bar{G}$ is a mapping from $G$ into $\bar{G}$ that preserves the group operation; that is, $(a b) \phi=(a \phi)(b \phi)$ for all $a, b$ in $G$.

Definition 3.

(Kernel of a Homomorphism) The Kernel of a homomorphism $\phi$ from a group $G$ to a group with identity $e$ is the $\operatorname{set}\{x \in G \mid x \phi=e\}$. The kernel of $\phi$ is denoted by $\operatorname{Ker} \phi$.

\section{Ring}

Definition 4.

A ring $\mathrm{R}$ is a set with two binary operations, addition $(a+b)$ and multiplication $(a b)$ such that for all $a, b, c$ in $\mathrm{R}$.

1. $a+b=b+a$.

2. $(a+b)+c=a+(b+c)$.

3. There is an element 0 in $\mathrm{R}$ such that $a+0=a$.

4. There is an element $-a$ in $R$ such that $a+(-a)=0$.

5. $a(b c)=(a b) c$.

6. $a(b+c)=a b+a c$ and $(b+c) a=b a+c a$.

So, a ring is an Abelian group under addition, also having an asscociative multiplication that is left and right distributive over addition. Note that multiplication need not be commutative. When it is, we say the ring is commutative. Also, a ring need not have an identity under multiplication. When a ring other than $\{0\}$ has an identity under multiplication, we say the ring has a unity (or identity). A nonzero element of a commutative ring with unity need not have a multiplicative inverse. When it does, we say it is a unit of the ring. Thus, $a$ is a unit if $a^{-1}$ exists.

The following terminology and notation is convenient. If $a$ and $b$ belong to a commutative ring $R$ and $a$ is nonzero, we say $a$ divides $b$ (or $a$ is a factor of $b$ ) and write $a \mid b$, if there exists an element $c$ in $R$ such that $b=a c$. If $a$ does not divide $b$, we write $a \times b$. 
Example 2.

The set $\mathbb{Z}$ of integers under ordinary addition and multiplication is a commutative ring with unity 1 . The units of $\mathbb{Z}$ are 1 and -1 .

\section{Integral Domain}

Definition 5.

A nonzero element $a$ in a commutative ring $R$ is called a zero-divisor if there is nonzero element $b$ in $R$ such that $a b=0$.

Definition 6.

A commutative ring with a unity is said to be an integral domain if it has no zero-divisors.

Example 3.

The ring of integers is an integral domain.

Definition 7.

A commutative ring with a unity is called a field if every nonzero element is a unit.

\section{Ideal}

Definition 8.

A subring $A$ of a ring $R$ is called a (two-sided) ideal of $R$ if for every $r \in R$ and every $a \in A$ both $r a$ and ar are in $A$.

So, a subring $A$ of a ring $R$ is an ideal of $R$ if $A$ "absorbs" elements from $R$; that is, if $r A \subseteq A$ and $A r \subseteq A$ for all $r \in R$.

An ideal $A$ of $R$ is called a proper ideal of $A$ if $A$ is a proper subset of $R$. In practise, one identifies ideals with the following test, which is an immediate consequence of the definition of ideal.

Theorem 1.

A nonempty subset $A$ of a ring $R$ is an ideal of $R$ if:

1. $a-b \in A$ whenever $a, b \in A$.

2. $r a$ and $a r$ are in $A$ whenever $a \in A$ and $r \in R$.

Example 4.

For any ring $R,\{0\}$ and $R$ are ideals of $R$. The ideal $\{0\}$ is called the trivial ideal. [4]

\section{Module}

A left $R$-module $M$ over the ring $R$ consists of an abelian group $(M,+)$ and an operation $R \times M$ $\rightarrow M$ (called scalar multiplication, usually just written by juxtaposition, i.e. as $r x$ for $r$ in $R$ and $x$ in $M$ ) such that

For all $r, s$ in $R, x, y$ in $M$, we have

1. $\quad r(x+y)=r x+r y$

2. $(r+s) x=r x+s x$

3. $(r s) x=r(s x)$

4. $1_{R} x=x$ if $R$ has multiplicative identity $1_{R}$.

If one writes the scalar action as $f_{r}$ so that $f_{r}(x)=r x$, and $f$ for the map which takes each $r$ to its corresponding map $f_{r}$, then the first axiom states that every $f_{r}$ is a group homomorphism of $M$, and the other three axioms assert that $f$ is a ring homomorphis $m$ from $R$ to the endomorphism ring $\operatorname{End}(M)$. Thus a module is a ring action on an abelian group (cf. group action. Also consider Monoid action of multiplicative structure of $\mathrm{R}$ ). In this sense, module theory 
generalizes representation theory, which deals with group actions on vector spaces, or equivalently group ring actions.

\section{Example 5.}

The set of rational numbers $\mathbb{Q}$ (for quotient) is a module $\mathrm{M}$.

Usually, we simply write "a left $R$-module $M$ " or ${ }_{R} M$. A right $R$-module $M$ or $M_{R}$ is defined similarly, only the ring acts on the right, i.e. we have a scalar multiplication of the form $M \times R$ $\rightarrow M$, and the above axioms are written with scalars $r$ and $s$ on the right of $x$ and $y$. (Authors who do not require rings to be unital omit condition 4 above in the definition of an $R$-module, and so would call the structures defined above "unital left $R$-modules". In this article, consistent with the glossary of ring theory, all rings and modules are assumed to be unital.)

A bimodule is a module which is a left module and a right module such that the two multiplications are compatible.

If $R$ is commutative, then left $R$-modules are the same as right $R$-modules and are simply called $R$-modules.

\section{Submodules \& Homomorphisms}

Suppose $M$ is a left $R$-module and $N$ is a subgroup of $M$. Then $N$ is a submodule (or $R$ submodule, to be more explicit) if, for any $n$ in $N$ and any $r$ in $R$, the product $r n$ is in $N$ (or $n r$ for a right module).

The set of submodules of a given module $M$, together with the two binary operations + and $\cap$, forms a lattice which satisfies the modular law: Given submodules $U, N_{1}, N_{2}$ of $M$ such that $N_{1}$ $\subset N_{2}$, then the following two submodules are equal: $\left(N_{1}+U\right) \cap N_{2}=N_{1}+\left(U \cap N_{2}\right)$.

If $M$ and $N$ are left $R$-modules, then a map $f: M \rightarrow N$ is a homomorphism of $R$-modules if, for any $m, n$ in $M$ and $r, s$ in $R: f(r m+s n)=r f(m)+s f(n)$

This, like any homomorphism of mathematical objects, is just a mapping which preserves the structure of the objects. Another name for a homomorphism of modules over $R$ is an $R$-linear map.

A bijective module homomorphism is an isomorphism of modules, and the two modules are called isomorphic. Two isomorphic modules are identical for all practical purposes, differing solely in the notation for the ir elements.

The kernel of a module homomorphism $f: M \rightarrow N$ is the submodule of $M$ consisting of all elements that are sent to zero by $f$. The is omorphism theorems familiar from groups and vector spaces are also valid for $R$-modules.

The left $R$-modules, together with their module homomorphisms, form a category, written as $R$ Mod. This is an abelian category [5].

\section{Left \& Right Modules}

In this section, we do not assume that the rings involved to be commutative.

Definition 9.

(Left modules) Let $\mathrm{R}$ be a ring. A left $\mathrm{R}$-modules (or a left module over) is an abelian group $\mathrm{M}$ together with a map $(a, m)$ of $R \times M$ into $M$ satisfying the following properties:

1) $a(m+n)=a m+a n$

2) $(a+b) m=a m+b m$

3) $(a b) m=a(b m)$

4) $1 m=m$

for $m, n \in M$ and $a, b \in R$. The map $R \times M \rightarrow M$ is refered to as the scalar multiplication.

This definition is somewhat familiar to us, as we can see in the following example of vector spaces.

Example 6.

If $R$ is a field, then a left $R$-module is what is known as the $K$-vector space. 
Example 7.

The abelian group

$R^{n}=R \times \ldots \times R$

$\underbrace{}_{\text {peopies }}$

is a left $R$-module if we left

$$
a\left(a_{1}, \ldots, a_{n}\right)=\left(a a_{1}, \ldots, a a_{n}\right),
$$

Where $a, a_{1}, \ldots, a_{n} \in R$. In particular, $R$ is a left $R$-module.

Example 8.

Let $A$ be a commutative ring and let $R$ be the ring of $m \times n$ matrices over $A$. Let $M$ be the abelian group of column $n$-vector in $A^{n}$. Then $M$ can be made into a left $R$-module by using the matrix multiplication as the scalar multiplication.

Proposition 1.

Let $M$ be a left $R$-module. then the followings are true:

1) $0_{R} m=0_{M}$

2) $a 0_{M}=0_{m}$

3) $(-a) m=-a m=a(-m)$ and in particular $(-1) m=-m$

Example 9.

Let $\mathrm{M}$ be a left $R$-module and let $B=\{b \in R: b m=0$ for all $m \in M\}$.

Then $B$ is a left ideal in $R$. Furthermore, if $I$ is any left ideal contained $B$ then $M$ becomes a left $R / I$-module by defining $(a+\mathrm{I}) m=a m$.

The notion of right $\mathrm{R}$-modules is dual to that of the left $\mathrm{R}$-modules.

Defintion 10.

(Right modules). Let $R$ be a ring. A right $R$-module (or a right module over $R$ ) is an abelian group $M$ together with a map $(m, a) \neg m a$ of $M \times R$ into $M$ satisfying the following properties:

1) $(m+n) a=m a+n a$

2) $m(a+b)=m a+m b$

3) $m(a b)=(m b) a$

4) $m 1=m$

for $m, n \in M$ and $a, b \in R$.

\section{Baer Modules}

Lee and Zhou introduced Baer, quazi-Baer and p.p. as follows modules:

1) $M_{R}$ is called Baer (respectively quazi-Baer) if, for any subset (respectively submodule) $X$ of $M, \operatorname{ann}_{R}(X)=e R$, where $e^{2}=e \in R$.

2) $M_{R}$ is called principally projective (or simply p.p.) module (respectively right principally quasi-Baer (or simply right p.q.-Baer) module) if, for any element $m \in M, a n n_{R}(m)=e R$ (resp. $\operatorname{ann}_{R}(m R)=e R$ ) where $e^{2}=e \in R$. [3]

\section{Semicommutative Modules}

Let $M$ be an $R$-module. Recall that $M$ is called semicommutative module if for any $a \in R$ and $m \in M, m a=0$ implies $m R a=0$ and $R$ is called a semicommutative ring if $R_{R}$ is a semicommutative module. in this work we will call $M S$-semicommutative if for any $f \in S$ and $m \in M, f(m)=0$ implies $f g(m)=0$ for every $g \in S$. Then a ring $R$ is a semicommutative ring if and only if $R_{R}$ is an $S$-semicommutative module where $S=\operatorname{End}_{R}\left(R_{R}\right) \cong R$. Note that any submodule $N$ of an $S$-semicommutative module $M$ is $S$-semicommutative. $M$ is $S$-principally quasi-Baer (or $S$-p.q.-Baer for short) if for any $m \in M, 1_{S}(m)=S e$ (which is equal to $1_{S}(m R)$ ) for some $e^{2}=e \in S$. A ring is called an abelian ring if its idempotents are central. And also note that if $M$ is an $S$-semicommutative module, then for all $\alpha \in S, \operatorname{Ker}(\alpha)$ is a fully 
invariant submodule of $M$ and so $M$ satisfies summand intersection property, that is, intersection of two direct summand of $M$ is again direct summand. [2]

\section{Reduced Modules}

A ring is reduced if it has no nonzero nilpotent (make zero) elements. A module $M$ is called reduced if, for any $m \in M$ and any $a \in R, m a=0$ implies $m R \cap M a=0$. [1]

\section{Reduced Modules Are Abelian}

Definition 11. A module $M$, is called abelian, if for any $m \in M$ and any $a \in R$, any idempotent $e \in R$, mae $=$ mea.

Lemma 1. If the module $M$ is semicommutative, then $M$ is abelian. The converse holds if $M$ is a p.p.-module.

Proof. Let $e$ be an idempotent in $R$ and $m \in M, a \in R$. Since $e$ is idempotent and $M$ is semicommutative, we have $m e\left(1_{R}-e\right)=0$ implies that $m e R\left(1_{R}-e\right)=0$. For any $a \in R$ we have $m e a\left(1_{R}-e\right)=0$, that is, mea = meae. On the orther hand, $m\left(1_{R}-e\right) e=0$ implies that $m\left(1_{R}-e\right) R e=0$. Then $m\left(1_{R}-e\right) a e=0$ and so mae = meae. Hence mea $=$ mae. Thus $\mathrm{M}$ is abelian. Suppose now $\mathrm{M}$ is abelian and p.p.-module. Let $m \in M$ and $a \in R$ with $m a=0$. Then $a \in r(m)=e R$ for some $e^{2}=e \in R$. So $m e=0$ and $a=e a$. Hence $m e R=0$. By the assumption $m R e=0$. Multiplying from the right by a, we have $m R e a=0$. Since $a=e a, m R a=0$. Thus $\mathrm{M}$ is semicommutative.

Lemma 2. if $M$ is a reduced module, then $M$ is abelian. The converse holds if $M$ is a.p.p.module.

Proof. Let $M$ be reduced. Since any reduced module is semicommutative and by Lemma 4.2., any semicommutative module is abelian, $M$ is abelian. Conversely, let $M$ be an abelian and p.p.module. Suppose $m a=0$ for $m \in M$ and $a \in R$. If $x \in m R \cap M a$, then there exists $m_{1} \in M$ and $r_{1} \in R$ such that $x=m r_{1}=m_{1} a$. Since $M$ is a p.p.-module, $m a=0$ implies that $a \in r_{R}(m)=$ $e R$ for some idempotent $e^{2}=e \in R$. Then $a=e a$ and $x e=m r_{1} e=m_{1} a e$. Since $M$ is abelian and $m e=0, m r_{1} e=m e r_{1}=m_{1} a e=m_{1} e a=m_{1} a=0$. So, $x=m r_{1}=m_{1} a=0$. Hence $m R \cap M a=0$, that is, $M$ is reduced.

\section{Conclusion}

If $x \in m R \cap M a$, then there exists $m_{1} \in M$ and $r_{1} \in R$ such that $x=m r_{1}=m_{1} a$. Since $M$ is a p.p.-module, $m a=0$ implies that $a \in r_{R}(m)=e R$ for some idempotent $e^{2}=e \in R$. Then $a=e a$ and $x e=m r_{1} e=m_{1} a e$. Since $M$ is abelian and $m e=0, m r_{1} e=m e r_{1}=$ $m_{1} a e=m_{1} e a=m_{1} a=0$. So, $x=m r_{1}=m_{1} a=0$. Hence $m R \cap M a=0$, that is, $M$ is reduced. Reduced modules are abelian.

\section{References}

[1] Agayev, N., G. Gungoroglu, A. Harmanci, and S. Halicioglu. 2009. Abelian Modules. Acta Math. Univ. Comen ianae.78: 235-244

[2] Agayev, N., T. Ozen and A. Harmanci. 2009. On Class of Se mico mmutative Modules. Proc. Indian Scad. Sci. (Math Sci). 119: 149-158

[3] Alhevaz, A. and A. Moussavi. 2010. On $\alpha$-Skew Quasi Armendariz Modules. Depart ment of Pure Mathematics, Faculty of Mathematical Sciences, Tarbiat Modares University: Tehran, Iran. 5: 23312340

[4] Gallian, Joseph A. 1990. Contemporary Abstract Algebra. D.C. Heath and Company: Lexington, Massachusetts Toronto

[5] Jacobson, Nathan. Structure of rings. 1964. Colloquiu m publications, Vol. 37, 2nd Ed., AMS Bookstore. ISBN 9780821810378 